\title{
Electrochemical determination of iron in coastal waters based on ionic liquid-reduced graphene oxide supported gold nanodendrites
}

\author{
Fei $\mathrm{Li}^{\mathrm{a}}$, Dawei Pan ${ }^{\mathrm{b}, *}$, Mingyue Lin ${ }^{\mathrm{b}}$, Haitao Han ${ }^{\mathrm{b}}$, Xueping $\mathrm{Hu}^{\mathrm{b}}$, Qi Kang ${ }^{\mathrm{a}, * *}$ \\ a The Key Lab in Molecular and Nano-materials Probes of the Ministry of Education of China, College of Chemistry, Chemical Engineering and Materials \\ Science, Shandong Normal University, Jinan, Shandong 250014, PR China \\ ${ }^{\mathrm{b}}$ Key Laboratory of Coastal Environmental Processes and Ecological Remediation, Yantai Institute of Coastal Zone Research (YIC), Chinese Academy of Sciences \\ (CAS), Shandong Provincial Key Laboratory of Coastal Environmental Processes, YICCAS, Yantai, Shandong 264003, PR China
}

\section{A R T I C L E I N F O}

\section{Article history:}

Received 13 May 2015

Received in revised form 30 June 2015

Accepted 3 July 2015

Available online 14 July 2015

\section{Keywords:}

Ionic liquid

Reduced graphene oxide

Gold nanodendrites

Iron

Coastal waters

\begin{abstract}
A B S T R A C T
An effective and sensitive method for electrochemical determination of iron was reported, based on the ionic liquid-reduced graphene oxide (IL-rGO) supported gold nanodendrites (AuNDs). IL-rGO as a soft support could provide large specific surface area for AuNDs and make them smaller sizes and unique forms, which would benefit to the electrochemical reduction of iron. Nafion is employed as a cation exchange polymer in which IL-rGO and AuNDs can be tightly attached to the electrode surface. The proposed sandwich structured IL-rGO/AuNDs/Nafion modified electrode shows excellent electrochemical properties. The IL-rGO/AuNDs/Nafion modified electrode combined individual advantages as a whole and showed good responses for iron ions. Under the optimized conditions, the reduction peak currents of iron have a good linear relation with its concentrations ranging from 0.30 to $100 \mu \mathrm{mol} \mathrm{L}^{-1}$ with the detection limit of $35 \mathrm{nmol} \mathrm{L}^{-1}$. More importantly, this sandwich structured modified electrode had a good anti-interference ability and successfully applied in the determination of the total dissolved iron in coastal waters.
\end{abstract}

(c) 2015 Elsevier Ltd. All rights reserved.

\section{Introduction}

Iron is widely distributed in nature and it is an essential nutrient and is abundant in many mineral oxides that exchange iron with surface and ground waters through dissolution and precipitation. Its most common oxidation states are $\mathrm{Fe}(\mathrm{II})$ and $\mathrm{Fe}$ (III); $\mathrm{Fe}(\mathrm{II})$ is a relatively soluble species in water, which is rapidly oxidized in oxygen rich environments. Conversely, Fe(III) forms strong complexes and is thermodynamically stable in water albeit of low solubility [1]. The concentration of soluble iron in natural water is significantly different than those in river water $(1 \mu \mathrm{mol}$ $\left.\mathrm{L}^{-1}\right)$, coastal water $\left(1 \mathrm{nmol} \mathrm{L}^{-1}\right)$, and ocean water $\left(10 \mathrm{pmol} \mathrm{L}^{-1}\right)[2]$. However, iron concentration can be higher in sediment pore waters. Iron can be determined by several methods such as inductively coupled plasma mass spectrometry (ICP-MS) [3], atomic absorption spectrometry (AAS) [4], cathodic stripping voltammetry (CSV) [5-7], luminescence [8], and spectrophotometry $[9,10]$, preceded sometimes by column preconcentration.

\footnotetext{
* Corresponding author. Fax: +865352109155.

** Corresponding author. Fax: +86 53182615258.

E-mail addresses: dwpan@yic.ac.cn (D. Pan), qikang@sdu.edu.cn (Q. Kang).
}

However, most of the sensitive and selective methods available are expensive to be used in routine analysis (ICP-MS and AAS). In fact, chemical modification of the electrode surface not only can lead to an increased electrocatalytic activity, but also better analytical selectivity and sensitivity is often achieved.

Nanomaterials play interesting roles in various fields of analytical chemistry [11-14]. In recent years, graphene has received considerable attention due to its high surface area $\left(2600 \mathrm{~m}^{2} \mathrm{~g}^{-1}\right)$, high chemical stability, excellent conductivity and strong mechanical strength. However, graphene sheets, unless well separated from each other, tend to form irreversible agglomerates or even restack to form graphite through van der Waals interactions. Graphene-nanoparticle composites have become a hot research topic in material science because the composite process can be an effective strategy to enhance their electronic, chemical, and electrochemical properties [15-17]. However, the lack of an efficient approach to produce polydisperse and longterm stable graphene sheets in different solvents has been a major obstacle to their exploitation in most of the proposed applications. Therefore, great efforts have been made to increase its solubility through the functionalization of graphene such as sulfonated graphene [18-20], using polymers or other molecules as functionalization reagents [21-24]. Among these functionalization 
reagents, ionic liquids (ILs) have attracted much attention because of their tunable structures and unique physicochemical properties such as wide electrochemical windows, high ionic conductivity, superior thermal stability, good solubility, and biocompatibility $[25,26]$. Furthermore, the introduction of IL moieties into functional graphene composites could not only increase their solubility, but also improve their performance.

Gold nanomaterials have the unique properties such as facilitating electron transfer between the species and electrode [27-29]. Gold nanostructures are more diverse, such as flowers [30] and rods [31]. Among them, there is an increasing interest for the fabrication of gold dendrites (AuNDs) [32]. Gold nanoparticals (AuNPs) is two-dimensional (2D) layer on the electrode surface. Compared to 2D AuNPs structure, AuNDs, which has a hyperbranched three-dimensional (3D) architecture, substantially enlarges the surface area of the electrode available for electrochemical detections.

In present work, ionic liquid-reduced graphene oxide (IL-rGO) was used as a support for electrochemical deposition of AuNDs. The resulting IL-rGO/AuNDs/Nafion modified electrode showed excellent electrochemical activities for iron ions. IL-rGO could provide large specific surface area for AuNDs and make it form smaller particles of the 3D structure, while AuNDs might facilitate electron transfer between the iron ions and electrode. Nafion was utilized as membrane matrix to improve the stability of electrode and accelerate the ion-exchange. The IL-rGO/AuNDs/Nafion modified electrode exhibited excellent selectivity, reproducibility and longterm stability for the determination of $\mathrm{Fe}(\mathrm{III})$ with lower detection limit and wider linear range. This novel sandwich structured IL-rGO/ AuNDs/Nafion modified electrode was also applied for the determination of total dissolved iron in real coastal waters.

\section{Experimental}

\subsection{Reagents}

Iron standard solution was purchased from Acros Organics. 1(3-aminopropyl)-3-methylimidazolium chloride ( $\mathrm{IL}-\mathrm{NH}_{2}$ ) was supplied by Shanghai Cheng Jie Chemical Co. Ltd. Graphene was supplied by Nanjing Jcnano Technology Co. Ltd. $\mathrm{HAuCl}_{4} \cdot 4 \mathrm{H}_{2} \mathrm{O}$ were purchased from Sinopharm Chemical Reagent Co. Ltd. Nafion (5\%) was purchased from Sigma Co. Ltd. All other chemicals were of analytical reagents and used without further purification. All experiments were conducted at room temperature, and the solutions were used in the process without nitrogen atmosphere.
Deionized water (18.2 $\mathrm{M} \Omega \mathrm{cm}$ specific resistance) obtained with a Pall Cascada laboratory water system was used throughout.

\subsection{Apparatus}

The morphology of the modified electrodes was characterized by using scanning electron microscopy (SEM Hitachi S-4800 microscope, Japan). All electrochemical experiments were carried out in a conventional three-electrode cell controlled by Electrochemical Work Station (CHI 660D, CH Instruments, Inc.). A modified glassy carbon (GC) disk ( $3 \mathrm{~mm}$ in diameter) was used as the working electrode, with $\mathrm{Ag} / \mathrm{AgCl}$ and platinum foil serving as the reference and counter electrodes, respectively. All potential values given below refer to $\mathrm{Ag} / \mathrm{AgCl}$.

\subsection{Synthesis of IL-rGO}

IL-rGO was synthesized by an epoxide ring-opening reaction between graphene oxide (GO) and the IL- $\mathrm{NH}_{2}$ according to the previous report [22]. Firstly, $\mathrm{IL}_{-} \mathrm{NH}_{2}(10 \mathrm{mg})$ was added into $10 \mathrm{~mL}$ of $\mathrm{GO}$ homogeneous dispersion in water $\left(0.5 \mathrm{mg} \mathrm{mL}^{-1}\right)$, Secondly, $\mathrm{KOH}$ (10 mg) was added into the above turbid mixture and then the mixture was subjected to ultrsonication for $30 \mathrm{~min}$. Finally, the homogeneous solution was vigorously stirred at $80^{\circ} \mathrm{C}$ for $24 \mathrm{~h}$. The resulting IL-rGO was subsequently centrifuged, washed with ethanol and water, dried under room temperature in the air, and re-dispersed in ultrapure water $\left(0.5 \mathrm{mg} \mathrm{mL}^{-1}\right)$.

\subsection{Preparation of the IL-rGO/AuNDs/Nafion modified electrode}

Prior to use, the GCE was polished using aqueous alumina slurry ( 0.3 and $0.05 \mu \mathrm{m}$ ), washed with deionized water thoroughly, and then ultrasonicated for $1 \mathrm{~min}$. The IL-rGO suspensions $(10 \mu \mathrm{L})$ were added on the surface of GCE and dried using an infrared lamp to get the IL-rGO composite modified electrode(GCE/IL-rGO). The modified electrode was then immersed in a $1.0 \mathrm{mmol} \mathrm{L}^{-1}$ chlorauric acid solution containing $0.2 \mathrm{~mol} \mathrm{~L}^{-1}$ of sodium sulfate to electrodeposit gold with constant potential at $-0.2 \mathrm{~V}$ for 60 s. Lastly, $6 \mu \mathrm{L}$ of $0.5 \%$ Nafion solution was dropped on the GCE/IL-rGO/AuNDs and dried at room temperature to form a uniformly coated electrode (GCE/ILrGO/AuNDs/Nafion). The obtained GCE/IL-rGO/AuNDs/Nafion was washed carefully with deionized water and then dried at room temperature. The schematic illustration of the stepwise selfassembly procedure is shown in Scheme 1. In order to compare with the electrochemical behavior for different electrodes, three

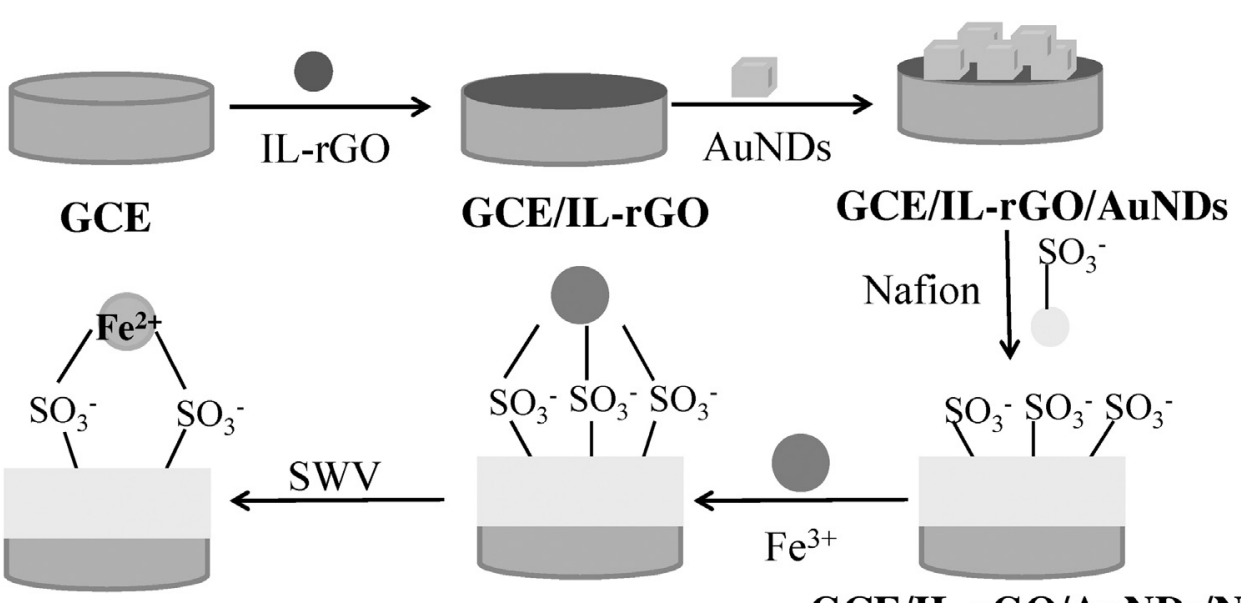

GCE/IL-rGO/AuNDs/Nafion

Scheme 1. The schematic illustration of the stepwise self-assembly procedure. 
modified electrodes, that is, GCE/Nafion, GCE/IL-rGO and GCE/IL-rGO/AuNDs were fabricated using a similar method.

\subsection{Analysis procedure}

Unless otherwise stated, the experiments were performed in the $0.1 \mathrm{~mol} \mathrm{~L}^{-1} \mathrm{HCl}$ electrolyte. CV and SWV methods were adopted. The parameters used in CV are as follows: initial potential of $-0.2 \mathrm{~V}$, final potential of $0.6 \mathrm{~V}$ and a scan rate of $100 \mathrm{mV} \mathrm{s}^{-1}$. The reduction responses of $\mathrm{Fe}(\mathrm{III})$ to $\mathrm{Fe}(\mathrm{II})$ on the bare and modified electrode were investigated by SWV, using the following parameters: initial potential of $0.65 \mathrm{~V}$, final potential of $0.2 \mathrm{~V}$, an amplitude of $0.025 \mathrm{~V}$, potential incremental of $0.001 \mathrm{~V}$, and an equilibrium time of $2 \mathrm{~s}$.

\subsection{Real sample pretreatment}

Sediments 1 and 2 were gained from the Bohai Sea (Shandong province, China) at different locations. The sample treatment was performed as follows: the sediments were centrifuged at $3000 \mathrm{rpm}$ for $15 \mathrm{~min}$ to separate the pore water. The pore water was then
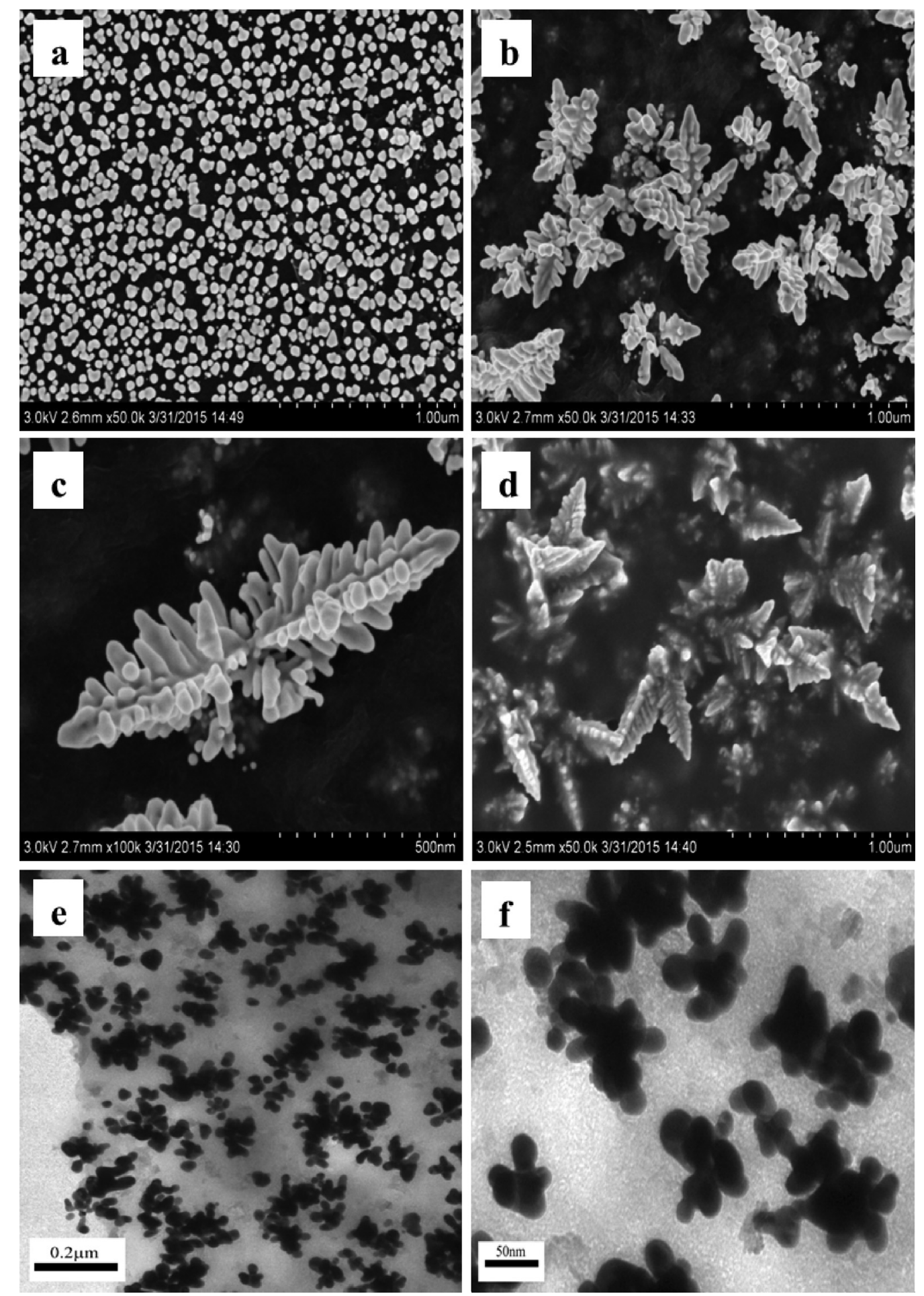

Fig. 1. SEM micrograph of the GCE/AuNPs (a), GCE/IL-rGO/AuNDs (b and c), GCE/IL-rGO/AuNDs/Nafion (d) and TEM micrograph of GCE/IL-rGO/AuNDs (e and f). 
acidified with $3 \mathrm{~mol} \mathrm{~L}^{-1} \mathrm{HNO}_{3}$ to adjust the $\mathrm{pH}$ less than 2.0 and kept at $4{ }^{\circ} \mathrm{C}$ until determination. Voltammetric measurements were performed on unfiltered samples diluted ten times of sediment pore waters with the $0.1 \mathrm{~mol} \mathrm{~L}^{-1} \mathrm{HCl}$ supporting electrolyte immediately prior to measurement. Coastal river water was collected from Guangdang river (a local coastal river, which flows into the Bohai Sea, Shandong province, China) and coastal sea water of Bohai Sea. All the water samples were collected in acid-cleaned polyethylene bottles after filtration $\left(0.45 \mu \mathrm{m}\right.$ membrane filters) and kept at $4{ }^{\circ} \mathrm{C}$ until determination. Voltammetric measurements were performed on water samples diluted ten times with the $0.1 \mathrm{~mol} \mathrm{~L}^{-1} \mathrm{HCl}$ supporting electrolyte immediately prior to measurement.

\section{Results and discussion}

\subsection{Characterization of the IL-rGO/AuNDs/Nafion modified electrode}

Fig. 1 shows the surface morphologies of GCE/AuNPs (Fig. 1a), GCE/IL-rGO/AuNDs (Fig. 1b and Fig. 1c), GCE/IL-rGO/AuNDs/Nafion (Fig. 1d) and TEM micrograph of GCE/IL-rGO/AuNDs (Fig. 1e and Fig. 1f). In Fig. 1a, the AuNPs were rough and non-uniform and the shape of them were spherical. Compared with Fig. 1a, Fig. 1b and Fig. 1c show plenty of AuNDs appeared in the IL-rGO film, which demonstrates a well ordered dendritic nanostructure. Fig. 1e and Fig. 1f further indicate the successful attachment of the AuNPs on the IL-rGO composite and the shape of $\mathrm{Au}$ is dendritic nanostructure. Considering the flakes structure of IL-rGO [22], the form of Au has been changed obviously. This is probably attributed to the slow crystal procedure in IL and the existence of rGO. IL-rGO has high viscosity and the structure of it is stereo. Au will be electrodeposited on IL-rGO and become dendrites on the surface of electrode. Additionally, in order to further prove that the nanodendrites were gold, GCE/IL-rGO/AuNDs was characterized by EDX (not shown). The EDX result showed that strong Au signals could be detected. Nanodendrites are highly branched with a well-defined 3D and globular structure. The SEM and TEM photographs illustrates that the IL-rGO is successfully modified on the surface of the GCE and have a well-defined morphology with almost uniform size and shape. Obviously, Fig. 1d shows that there is a layer of mulch on the surface of AuNDs compared with Fig. 1b. The SEM results indicate sandwich structured IL-rGO/AuNDs/Nafion are successfully modified on the surface of the GCE.

The active surface areas of the modified electrodes were estimated according to the slope of the Ip versus $v^{1 / 2}$ plot for a known concentration of $\mathrm{K}_{3} \mathrm{Fe}(\mathrm{CN})_{6}$, based on the Randles-Sevcik equation:

Ip $=2.69 \times 10^{5} n^{3 / 2} \mathrm{AD}^{1 / 2} v^{1 / 2} c_{0}$

Where Ip refers to the anodic peak current. $n$ is the electron transfer number. A is the surface area of the electrode. $D$ is the diffusion coefficient. $c_{0}$ is the concentration of $\mathrm{K}_{3} \mathrm{Fe}(\mathrm{CN})_{6}$ and $v$ is the scan rate. For $1.0 \mathrm{mmol} \mathrm{L}^{-1} \mathrm{~K}_{3} \mathrm{Fe}(\mathrm{CN})_{6}$ in $0.10 \mathrm{~mol} \mathrm{~L}^{-1} \mathrm{KCl}$ electrolyte, $n$ equals 1 and $\mathrm{D}$ is $7.6 \times 10^{-6} \mathrm{~cm} \mathrm{~s}^{-1}$. The active surface areas were calculated from the slope of the Ip vs. $v^{1 / 2}$. Thus, the surface areas of GCE/IL-rGO/AuNDs, GCE/IL-rGO and GCE are 0.11, 0.097 and $0.061 \mathrm{~cm}^{2}$, respectively. The results showed that the presence of IL-rGO and AuNDs can cause the increase of the active surface of the electrode obviously.

The typical CVs for GCE/IL-rGO/AuNDs/Nafion, GCE/IL-rGO/AuNDs, GCE/IL-rGO and bare GCE were recorded between $-0.2 \mathrm{~V}$ and $0.6 \mathrm{~V}$ in $1 \mathrm{mmol} \mathrm{L}^{-1} \mathrm{Fe}(\mathrm{CN})_{6}{ }^{3-/ 4-}$ and $0.1 \mathrm{~mol} \mathrm{~L}^{-1} \mathrm{KNO}_{3}$ solution (Fig. 2). Quasi-reversible one-electron redox behavior of $\mathrm{Fe}(\mathrm{CN})_{6}{ }^{3-14-}$ was observed on the bare GCE (Fig. 2a), with a peak separation $(\triangle E p)$ of $83 \mathrm{mV}$ and peak current (Ip) was $10.1 \mu \mathrm{A}$. After the electrode was modified with IL-rGO

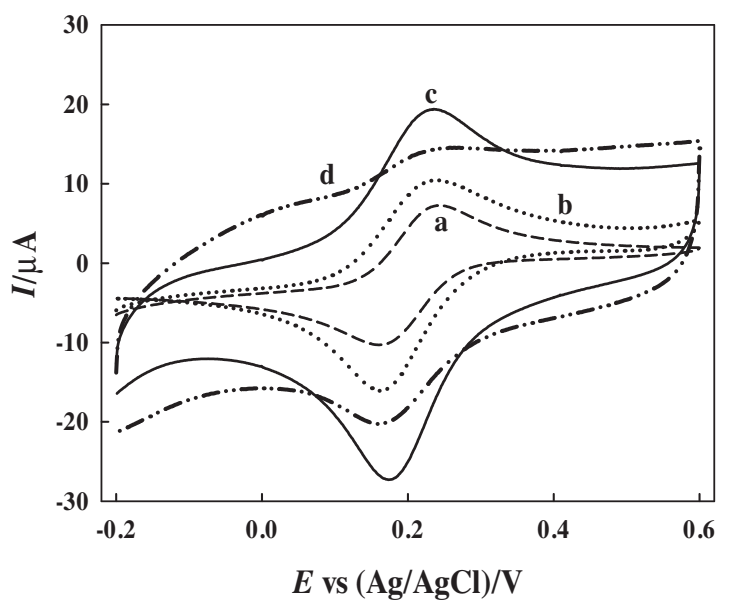

Fig. 2. CVs of bare GCE (a), GCE/IL-rGO (b), GCE/IL-rGO/AuNDs (c) and GCE/IL-rGO/AuNDs/Nafion (d) in $1 \mathrm{mmol} \mathrm{L}^{-1}\left[\mathrm{Fe}(\mathrm{CN})_{6}\right]^{3-/ 4-}$ and $0.1 \mathrm{~mol} \mathrm{~L}^{-1} \mathrm{KCl}$ solution in the potential range of -0.2 to $0.6 \mathrm{~V}$. Scan rate: $50 \mathrm{mV} \mathrm{s}^{-1}$.

(Fig. 2b), I $p$ was increased and $\Delta E p$ was decreased compared with the bare GCE, indicating that IL-rGO has a catalytically active surface and a very high aspect ratio. For the deposition of AuNDs on the GCE/IL-rGO (Fig. 2c), Ip was increased and $\Delta E p$ was decreased compared with that of the IL-rGO modified electrode, indicating that AuNDs play a role in the increase of the electroactive surface area. However, the Ip of the modified IL-rGO/AuNDs/Nafion electrode (Fig. 2d) was decreased compared with that of the bare GCE, due mainly to the blocking behavior of Nafion and it is negatively charged and hinders the diffusion of $\mathrm{Fe}(\mathrm{CN})_{6}{ }^{3-/ 4-}$ toward the electrode surface.

\subsection{Electrochemical behavior of iron on the IL-rGO/AuNDs/Nafion modified electrode}

Fig. 3 shows the SWVs of GCE/Nafion (Fig. 3a), GCE/IL-rGO/AuNDs (Fig. 3b) GCE/AuNPs/Nafion (Fig. 3c) and GCE/IL-rGO/AuNDs/Nafion (Fig. 3d) in $0.1 \mathrm{~mol} \mathrm{~L}^{-1} \mathrm{HCl}$ solution containing $5 \mathrm{mmol} \mathrm{L}^{-1} \mathrm{Fe}(\mathrm{III})$. The small reduction peak of $\mathrm{Fe}(\mathrm{III})$ was obtained at GCE/Nafion, GCE/IL-rGO/AuNDs and GCE/AuNPs/Nafion. The peak potential of iron is $0.43 \mathrm{~V}, 0.45 \mathrm{~V}$ and $0.43 \mathrm{~V}$, the peak current of iron is $0.30 \mu \mathrm{A}$, $0.46 \mu \mathrm{A}$ and $0.85 \mu \mathrm{A}$ (Fig. 3a, Fig. $3 \mathrm{~b}$ and Fig. $3 \mathrm{c}$ ), respectively. While the peak potential of iron at GCE/IL-rGO/AuNDs/Nafion is $0.43 \mathrm{~V}$ and the peak current is $2.43 \mu \mathrm{A}$ (Fig. $3 \mathrm{~d}$ ). The peak current of iron at the GCE/IL-rGO/AuNDs/Nafion showed up to the 7-fold, 4-fold and

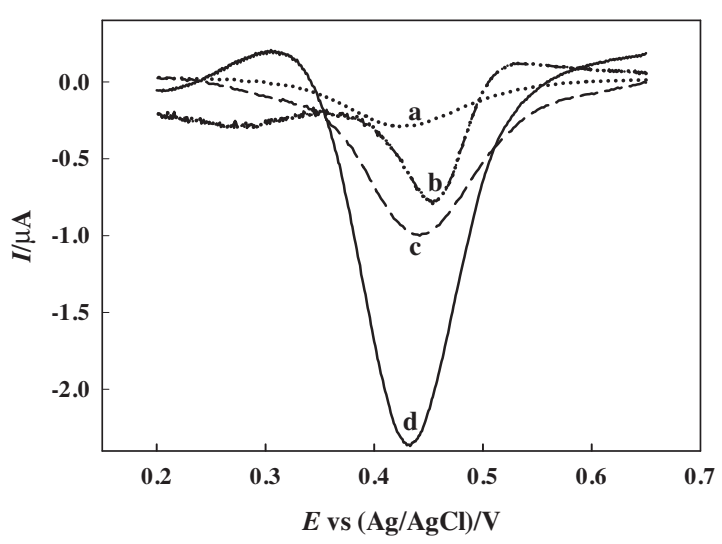

Fig. 3. SWVs of GCE/Nafion (a), GCE/IL-rGO/AuNDs (b), GCE/AuNPs/Nafion (c) and GCE/IL-rGO/AuNDs/Nafion (d) in $0.1 \mathrm{~mol} \mathrm{~L}^{-1} \mathrm{HCl}$ containing $5 \mu \mathrm{mol} \mathrm{L}^{-1}$ iron. Scan rate: $25 \mathrm{mV} \mathrm{s}^{-1}$. 
3-fold improvement than that at GCE/Nafion, GCE/IL-rGO/AuNDs and GCE/AuNPs/Nafion. As a result of the catalysis of AuNDs, the reduction peak potential of iron shifted positively suggesting that the AuNDs not only possess strong electrocatalytic activity toward the redox reaction but also promoted the electron-transfer rate on the surface of the electrode. IL not only works as reducing agent for GO but also makes the crystal procedure of Au slowly due to its high viscosity. IL-rGO has high electronic, chemical, electrochemical properties, provides large specific surface area for AuNDs and makes them smaller sizes and unique forms, which would benefit to the electrochemical reduction of iron. Nafion is employed as a cation exchange polymer in which IL-rGO and AuNDs can be tightly attached to the electrode surface.

To obtain the kinetic parameters of $\mathrm{Fe}(\mathrm{III})$ at the sandwich structured IL-rGO/AuNDs/Nafion modified GCE, the relationship between scan rate and the peak current of iron was also investigated by linear sweep voltammetry. Fig. 4 shows that the cathodic peak current increased linearly with the increasing scan rate from 25 to $300 \mathrm{mV} \mathrm{s}^{-1}$. This result indicated that the electro-reduction reactions of iron at the GCE/IL-rGO/AuNDs/Nafion are typical adsorptioncontrolled processes. The determination mechanism of $\mathrm{Fe}(\mathrm{III})$ on GCE/IL-rGO/AuNDs/Nafion is shown in Scheme 1.

\subsection{Optimization for iron measurement on IL-rGO/AuNDs/Nafion modified electrode}

\subsubsection{Effect of the amount of the IL-rGO}

The amount of the IL-rGO composite at the electrode surface was investigated at the volumes ranging from 6.0 to $14.0 \mu \mathrm{L}$ (Fig. 5). The cathodic peak current increased gradually from 6.0 to $10.0 \mu$ L volume of the composite. The maximum current was observed at a volume of $10.0 \mu \mathrm{L}$. Then, the cathodic peak current decreased with increasing volume from 10.0 to $14.0 \mu \mathrm{L}$ because the redundant IL-rGO may hinder the electron transfer between Fe(III) and the electrode. The composite could not cover the electrode surface, and the modifier was uneven at volumes less than $10.0 \mu \mathrm{L}$. Thus, $10.0 \mu \mathrm{L}$ volume of the composite was considered as the optimal film thickness.

\subsubsection{Effect of the deposition time of the AuNDs}

The deposition time of the AuNDs at the GCE/IL-rGO surface was investigated at the time ranging from 30 s to 80 s (Fig. 6). The cathodic peak current gradually increased from 30 s to $60 \mathrm{~s}$. The maximum current was observed at the deposition time of $60 \mathrm{~s}$. But the cathodic peak current decreased with increasing deposition time from 60s to 80s because the size of AuNDs became bigger and

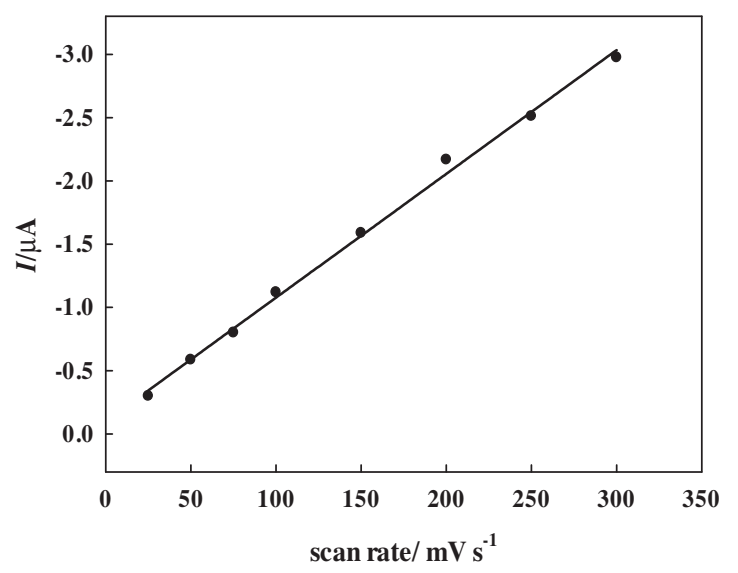

Fig. 4. Linear relationship of scan rate (from $25 \mathrm{mV} \mathrm{s}^{-1}$ to $300 \mathrm{mV} \mathrm{s}^{-1}$ ) versus reduction current response of $10 \mu \mathrm{mol} \mathrm{L}^{-1}$ iron in $0.1 \mathrm{~mol} \mathrm{~L}^{-1} \mathrm{HCl}$.

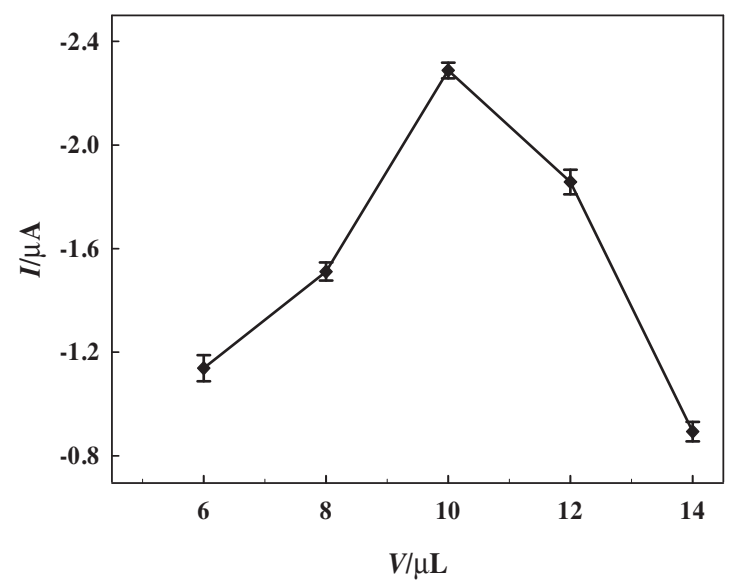

Fig. 5. Effect of the amount of the IL-rGO on the reduction current response of $5 \mu \mathrm{mo} \mathrm{L}^{-1}$ iron in $0.1 \mathrm{~mol} \mathrm{~L}^{-1} \mathrm{HCl}$ on GCE/IL-rGO/AuNDs/Nafion.

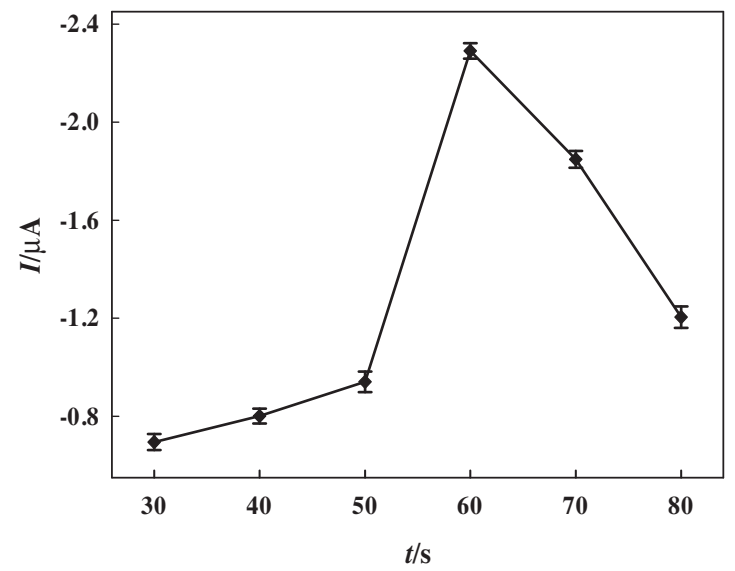

Fig. 6. Effect of the deposition time of the AuNDs on the reduction current response of $5 \mu \mathrm{mol} \mathrm{L}^{-1}$ iron in $0.1 \mathrm{~mol} \mathrm{~L}^{-1} \mathrm{HCl}$ on GCE/IL-rGO/AuNDs/Nafion.

the specific surface area decreased. Thus, 60s of the deposition time of AuNDs was considered as the optimal deposition time.

\subsubsection{Effect of the amount of Nafion solution}

The effect of the amount of Nafion at the GCE/IL-rGO/AuNDs surface was investigated at the volumes ranging from 2.0 to $10.0 \mu \mathrm{L}$

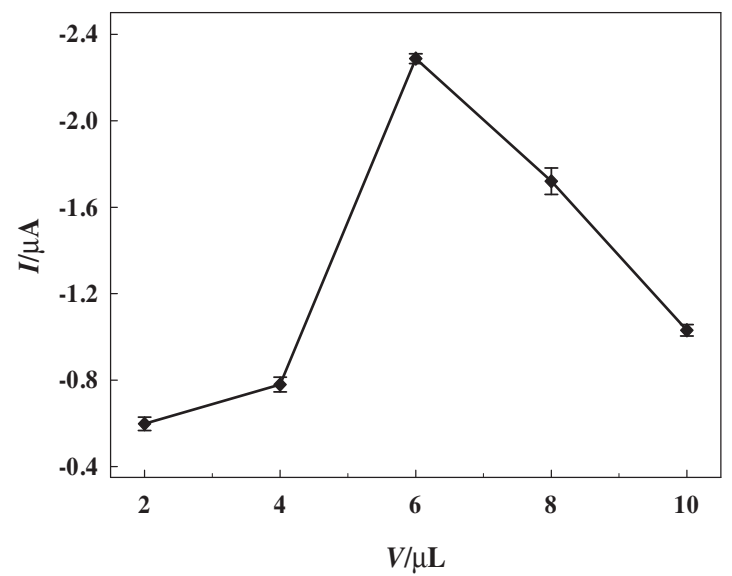

Fig. 7. Effect of the amount of Nafion solution on the reduction current response of $5 \mu \mathrm{mol} \mathrm{L}^{-1}$ iron in $0.1 \mathrm{~mol} \mathrm{~L}^{-1} \mathrm{HCl}$ on GCE/IL-rGO/AuNDs/Nafion. 


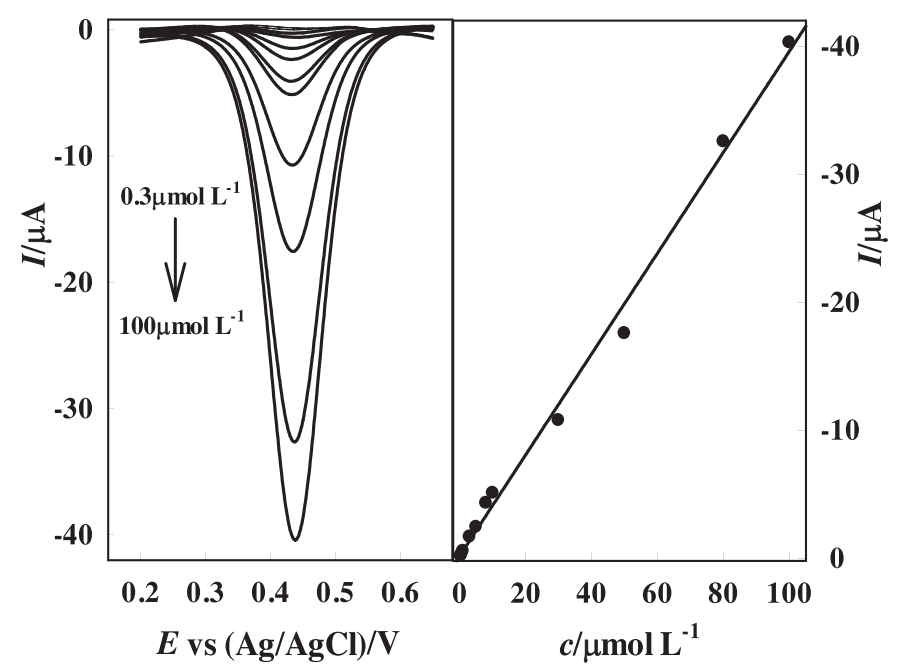

Fig. 8. The calibration curve for iron determination. Left: SWVs of the GCE/IL-rGO/ AuNDs/Nafion at different iron concentrations (from $0.3 \mu \mathrm{mol} \mathrm{L}^{-1}$ to $100 \mu \mathrm{mol} \mathrm{L}^{-1}$ ) in $0.1 \mathrm{~mol} \mathrm{~L}^{-1} \mathrm{HCl}$. Scan rate: $25 \mathrm{mV} \mathrm{s}^{-1}$.

(Fig. 7). The cathodic peak of iron current gradually increased from 2.0 to $6.0 \mu \mathrm{L}$ of the Nafion solution. The maximum current was observed at a volume of $6.0 \mu \mathrm{L}$. However, the cathodic peak current decreased with increasing volume from 6.0 to $10.0 \mu \mathrm{L}$ because the redundant Nafion may hinder the electron transfer between $\mathrm{Fe}(\mathrm{III})$ and the AuNDs. The Nafion could not cover the electrode surface, and the modifier was uneven at volumes less than $6.0 \mu \mathrm{L}$. Thus, $6.0 \mu \mathrm{L}$ of the Nafion solution was considered as the optimal amount.

\subsection{Calibration Curve}

The calibration curve of the Fe(III) was derived from the SWVs obtained at the GCE/IL-rGO/AuNDs/Nafion in $0.1 \mathrm{~mol} \mathrm{~L}^{-1} \mathrm{HCl}$ under the optimal conditions (Fig. 8). The peak current increased linearly in the $\mathrm{Fe}(\mathrm{III})$ concentration ranging from 0.3 to $100 \mu \mathrm{mol} \mathrm{L}^{-1}$. The equation for linear regression was expressed as follows:

Ip $=-0.394 c-0.204\left(0.3-100 \mu \mathrm{mol} \mathrm{L}^{-1}, \mathrm{R}^{2}=0.995\right)$

The sensitivity and detection limit of the GCE/IL-rGO/AuNDs/ Nafion for Fe(III) determination were $0.39 \mathrm{~A} \mathrm{~mol}^{-1} \mathrm{~L}$ and $35 \mathrm{nmol}$ $\mathrm{L}^{-1}(\mathrm{~S} / \mathrm{N}=3)$, respectively. Additionally, comparisons about iron determination by electrochemical and other analytical methods were presented in Table 1 . The electrochemical methods showed relatively superior performances, such as simple procedure, cheap instrument, low testing cost, relatively lower detection limit and a good linear range without using chelating agents. Above all, the GCE/IL-rGO/AuNDs/Nafion may be a good choice for iron determination.

\subsection{Reproducibility, repeatability and selectivity}

The reproducibility of GCE/IL-rGO/AuNDs/Nafion was investigated in $5 \mu \mathrm{mol} \mathrm{L}^{-1} \mathrm{Fe}$ (III) by ten independently modified electrodes prepared with the same method and the corresponding relative standard deviation (RSD) was 5.5\%, which indicated that there was no significant difference among the analytical signals between the prepared electrodes. The repeatability of the developed method was also evaluated by detecting $5 \mu \mathrm{mol} \mathrm{L}^{-1}$ $\mathrm{Fe}(\mathrm{III})$ using the same electrode for 10 measurements with RSD was 3.9\%. Hence, the GCE/IL-rGO/AuNDs/Nafion showed good reproducibility and repeatability.

The oxygen present in the solution was helpful to the detection because it can relatively accelerate the redox of $\mathrm{Fe}(\mathrm{III}) / \mathrm{Fe}(\mathrm{II})$. Moreover, the selectivity of the GCE/IL-rGO/AuNDs/Nafion for the iron determination was investigated by adding various foreign species into $0.1 \mathrm{~mol} \mathrm{~L}^{-1} \mathrm{HCl}$ containing $5 \mu \mathrm{mol} \mathrm{L}^{-1} \mathrm{Fe}(\mathrm{III})$. The 500 fold $\mathrm{Na}^{+}, \mathrm{K}^{+}, 300$-fold $\mathrm{Ca}^{2+}, 100$-fold $\mathrm{Mn}^{2+}, \mathrm{Cd}^{2+}, 50$-fold $\mathrm{Zn}^{2+}, \mathrm{Mg}^{2+}$, $\mathrm{Al}^{3+}, 10$-fold $\mathrm{Cr}^{3+}, 5$-fold $\mathrm{Cu}^{2+}, \mathrm{Pb}^{2+}$ did not affect the determination

Table 1

Comparison of the electrochemical methods and other analytical methods for iron determination.

\begin{tabular}{|c|c|c|c|c|c|}
\hline Methods & Electrode/agents & Linear range $\left(\mathrm{nmol} \mathrm{L}^{-1}\right)$ & Detecting limit $\left(\mathrm{nmol} \mathrm{L}^{-1}\right)$ & Sensitivity $\left(\mathrm{A} \mathrm{mol}^{-1} \mathrm{~L}\right)$ & References \\
\hline ICP-MS & nitrilotriacetic acid chelating resin & $0.04-10$ & $0.02-0.07$ & Not mentioned & [3] \\
\hline CSV & mercury coated, gold, micro-wire electrode & Not mentioned & 0.1 & Not mentioned & [5] \\
\hline $\mathrm{DPV}^{\mathrm{a}}$ & $\begin{array}{l}\text { rGO/MB/AuNPs }{ }^{\mathrm{b}} \text { composite modified } \\
\text { electrode }\end{array}$ & $300-100,000$ & 15 & 0.034 & [33] \\
\hline DLLME $^{\mathrm{C}} / \mathrm{UV}$-vis & 5-Br-PADAP ${ }^{\mathrm{d}}$ and ascorbic acid & $90-7000$ & 27 & Not mentioned & {$[10]$} \\
\hline CSV & $\mathrm{CMGCE}^{\mathrm{e}}$ & $357-5,357$ & 178 & Not mentioned & [34] \\
\hline SWV & EPPG $^{\text {f }}$ electrode & Not mentioned & 2000 & 0.06 & [35] \\
\hline SWV & $\begin{array}{l}\text { GCE/IL-rGO/AuNDs/ } \\
\text { Nafion }\end{array}$ & $300-100,000$ & 35 & 0.39 & Present work \\
\hline
\end{tabular}

a Differential pulse voltammetry.

b Reduced grapheme oxide/Methylene Blue/gold nanoparticles.

c Dispersive liquid-liquid microextraction.

d 2-(5-bromo-2-pyridylazo)-5-(diethyl amino) phenol.

e Chitosan modified glassy carbon electrode.

${ }^{\mathrm{f}}$ Edge plane pyrolytic graphite.

Table 2

Comparison of this method and ICP-MS for determination of total dissolved iron in real sediment pore waters and coastal waters ( $\mathrm{n}=3$ ).

\begin{tabular}{|c|c|c|c|c|}
\hline Samples & Iron added $\left(\mu \mathrm{mol} \mathrm{L}^{-1}\right)$ & $\begin{array}{l}\text { Detected by GCE/IL-rGO/AuNDs/ } \\
\text { Nafion }\end{array}$ & Detected by ICP-MS $\left(\mathrm{mg} \mathrm{g}^{-1}\right)$ & Recovery(\%) \\
\hline & & $\mathrm{mg} \mathrm{g}^{-1}$ & & \\
\hline sediment pore water 1 & 0 & $27.6 \pm 0.36$ & $28.0 \pm 0.20$ & - \\
\hline sediment pore water 2 & 0 & $\begin{array}{l}19.7 \pm 0.24 \\
\mu \mathrm{mol} \mathrm{L}^{-1}\end{array}$ & $20.0 \pm 0.14$ & - \\
\hline Coastal river water & 10.0 & $10.3 \pm 0.21$ & - & 103.2 \\
\hline Coastal sea water ${ }^{\mathrm{a}}$ & 5.0 & $4.9 \pm 0.30$ & - & 97.1 \\
\hline
\end{tabular}

a The salinity is $14.8 \%$. 
of iron ( $<5 \%$ of response current change). These results indicated excellent selectivity of the proposed electrode.

\subsection{Practical application of IL-rGO/AuNDs/Nafion modified electrode}

To evaluate the practical application of the established electrode, the GCE/IL-rGO/AuNDs/Nafion was used for the total dissolved iron determination in coastal water samples. The concentration of iron was estimated by standard addition method. The calculated result of sediment pore water samples were in agreement with the value detected by ICP-MS and the comparison results were displayed in Table 2 . Obviously, the results were consistent with ICP-MS detection, indicating that the fabricated electrode may function as a new and functional electrochemical sensor for the total dissolved iron determination in waters from coastal zone area.

\section{Conclusions}

In summary, a novel and effective sandwich structured IL-rGO/AuNDs/Nafion modified electrode was fabricated for sensitive determination of $\mathrm{Fe}(\mathrm{III})$. Compared to the GCE/Nafion and GCE/IL-rGO/AuNDs, the obtained IL-rGO/AuNDs/Nafion modified GCE enhances significantly the reduction signal of Fe(III) with a detection limit of $35 \mathrm{nmol} \mathrm{L}^{-1}$. Moreover, the modified electrode showed excellent reproducibility, repeatability and selectivity for determination of total dissolved iron in coastal water samples. The fabricated electrode may function as a novel electrochemical sensor for determination of iron and is a promising platform for accurate electroanalysis of trace iron.

\section{Acknowledgements}

This work was financially supported by the National Natural Science Foundation of China (41276093 and 21275091), the Youth Innovation Promotion Association (2011170) and Outstanding Young Scientists of CAS.

\section{References}

[1] X.W. Liu, F.J. Millero, The solubility of iron in seawater, Mar. Chem. 77 (2002) 43.

[2] M. Lu, N.V. Rees, A.S. Kabakaev, R.G. Compton, Determination of iron: electrochemical methods, Electroanalysis 24 (2012) 1693.

[3] J. de Jong, V. Schoemann, D. Lannuzel, J.L. Tison, N. Mattielli, High-accuracy determination of iron in seawater by isotope dilution multiple collector inductively coupled plasma mass spectrometry (ID-MC-ICP-MS) using nitrilotriacetic acid chelating resin for preconcentration and matrix separation, Anal. Chim. Acta 623 (2008) 126.

[4] F. Vignola, D.L.G. Borges, A.J. Curtius, B. Welz, H. Becker-Ross, Simultaneous determination of $\mathrm{Cd}$ and $\mathrm{Fe}$ in sewage sludge by high-resolution continuum source electrothermal atomic absorption spectrometry with slurry sampling, Microchem. J. 95 (2010) 333.

[5] J. Gun, P. Salaun, C.M.G. van den Berg, Advantages of using a mercury coated, micro-wire, electrode in adsorptive cathodic stripping voltammetry, Anal. Chim. Acta 571 (2006) 86.

[6] E.M. Ghoneim, Simultaneous determination of $\mathrm{Mn}(\mathrm{II}), \mathrm{Cu}(\mathrm{II})$ and $\mathrm{Fe}(\mathrm{III})$ as 2(5-bromo-2-pyridylazo)-5-diethylaminophenol complexes by adsorptive cathodic stripping voltammetry at a carbon paste electrode, Talanta 82 (2010) 646.

[7] M. Cheize, G. Sarthou, P.L. Croot, E. Bucciarelli, A.C. Baudoux, A.R. Baker, Iron organic speciation determination in rainwater using cathodic stripping voltammetry, Anal. Chim. Acta 736 (2012) 45.

[8] H. Obata, H. Karatani, E. Nakayama, Automated determination of iron in seawater by chelating resin concentration and chemiluminescence detection, Anal. Chem. 65 (1993) 1524.

[9] A.S. Amin, A.A. Gouda, Utility of solid-phase spectrophotometry for determination of dissolved iron(II) and iron(III) using 2,3-dichloro-6-(3carboxy-2-hydroxy-1-naphthylazo) quinoxaline, Talanta 76 (2008) 1241.
[10] B. Peng, Y.P. Shen, Z.T. Gao, M. Zhou, Y.J. Ma, S.G. Zhao, Determination of total iron in water and foods by dispersive liquid-liquid microextraction coupled with microvolume UV-vis spectrophotometry, Food Chem. 176 (2015) 188.

[11] A.K. Das, R.K. Layek, N.H. Kim, J. Samdani, M.C. Kang. J.H. Lee, Nicotinamide adenine dinucleotide assisted shape-controlled synthesis of catalytically active raspberry-like gold nanostructures, Electrochim. Acta 151 (2015) 195.

[12] H. Fan, P. Ju, S.Y. Ai, Controllable synthesis of CdSe nanostructures with tunable morphology and their application in DNA biosensor of Avian Influenza Virus, Sens. Actuators B 149 (2010) 98.

[13] H. Fan, S.X. Zhang, P. Ju, H.C. Su, S.Y. Ai, Flower-like Bi2Se $\mathrm{Se}_{3}$ nanostructures: Synthesis and their application for the direct electrochemistry of hemoglobin and $\mathrm{H}_{2} \mathrm{O}_{2}$ detection, Electrochim. Acta 64 (2012) 171.

[14] J.M. Macak, H. Tsuchiya, A. Ghicov, K. Yasuda, R. Hahn, S. Baure, P. Schmuki, $\mathrm{TiO}_{2}$ nanotubes: Self-organized electrochemical formation, properties and applications, Curr. Opin. Solid St. M 11 (2007) 3.

[15] H.C. Tian, J.Q. Liu, D.X. Wei, X.Y. Kang, C. Zhang, J.C. Du, B. Yang, X. Chen, H.Y. Zhu, Y.N. NuLi, Graphene oxide doped conducting polymer nanocomposite film for electrode-tissue interface, Biomaterials 35 (2014) 2120.

[16] M. Deng, X. Yang, M. Silke, W.M. Qiu, M.S. Xu, G. Borghs, H.Z. Chen, Electrochemical deposition of polypyrrole/graphene oxide composite on microelectrodes towards tuning the electrochemical properties of neural probes, Sens. Actuators B 158 (2011) 176

[17] S.C. Wang, J. Yang, X.Y. Zhou, J. Xie, L.L. Ma, B. Huang, Electrochemical properties of carbon nanotube/graphene oxide hybrid electrodes fabricated via layer-by-layer self-assembly, J. Electroanal. Chem. 722 (2014) 141.

[18] S.J. Li, Y.F. Shi, L. Liu, L.X. Song, H. Pang, J.M. Du, Electrostatic self-assembly for preparation of sulfonated graphene/gold nanoparticle hybrids and their application for hydrogen peroxide sensing, Electrochim. Acta 85 (2012) 628

[19] S.J. Li, J.Z. He, M.J. Zhang, R.X. Zhang, X.L. Lv, S.H. Li, H. Pang, Electrochemical detection of dopamine using water-soluble sulfonated graphene, Electrochim. Acta 102 (2013) 58.

[20] S.J. Li, G.Y. Zhao, R.X. Zhang, Y.L. Hou, L. Liu, H. Pang, A sensitive and selective nitrite sensor based on a glassy carbon electrode modified with gold nanoparticles and sulfonated grapheme, Microchim. Acta 180 (2013) 821.

[21] S. Radhakrishnan, K. Krishnamoorthy, C. Sekar, J. Wilson, S.J. kim, A promising electrochemical sensing platform based on ternary composite of polyaniline$\mathrm{Fe}_{2} \mathrm{O}_{3}$-reduced graphene oxide for sensitive hydroquinone determination, Chem. Eng. J. 259 (2015) 594.

[22] H.F Yang, C.S. Shan, FH, Li, D. Han, QX Zhang L Niu, Covalent functionalization of polydisperse chemically-converted grapheme sheets with amine-terminated ionic liquid, Chem. Commun. 26 (2009) 3880.

[23] M.L. Wang, Y.Q. Gao, J.J. Zhang, J.W. Zhao, Highly dispersed carbon nanotube in new ionic liquid-graphene oxides aqueous dispersions for ultrasensitive dopamine detection, Electrochim. Acta 155 (2015) 236.

[24] S.H. Zhang, X.M. Chen, G.S. Liu, X.D. Hou, Y.N. Huang, J.P. Chen, G.Q. Zhan, C.Y. Li, A novel sensing platform based on ionic liquid integrated carboxylicfunctionalized graphene oxide nanosheets for honokiol determination, Electrochim. Acta 155 (2015) 45.

[25] S.J. Yoo, L.J. Li, C.C. Zeng, R.D. Little, Polymeric Ionic Liquid and Carbon Black Composite as a Reusable Supporting Electrolyte: Modification of the Electrode Surface, Angew. Chem. Int. Edit. 54 (2015) 3744.

[26] L. Timperman, A. Vigeant, M. Anouti, Eutectic mixture of Protic Ionic Liquids as an Electrolyte for Activated Carbon-Based Supercapacitors, Electrochim. Acta 155 (2015) 164

[27] C.S. Shan, H.F. Yang, D.X. Han, Q.X. Zhang, A. Ivaska, L. Niu, Graphene/AuNPs/ chitosan nanocomposites film for glucose biosensing, Biosens. Bioelectron. 25 (2010) 1070.

[28] Y.Zhao, L.Z. Fan, J.L. Ren, B. Hong, Gold nanoclusters electrodeposited on multiwalled carbon nanotubes: enhanced electrocatalytic activity of hemoglobin, J Solid State Electrochem. 18 (2014) 1099.

[29] Y.R. An, X.L. Jiang, W.J. Bi, H. Chen, L.T. Jin, S.P. Zhang, C.G. Wang, W. Zhang, Sensitive electrochemical immunosensor for alpha-synuclein based on dual signal amplification using PAMAM dendrimer-encapsulated $\mathrm{Au}$ and enhanced gold nanoparticle labels, Biosens. Bioelectron. 32 (2012) 224.

[30] L. Zhong, X.D. Zhai, X.F. Zhu, P.P. Yao, M.H. Liu, Vesicle-Directed Generation of Gold Nanoflowers by Gemini Amphiphiles and the Spacer-Controlled Morphology and Optical Property, Langmuir 26 (2010) 5876.

[31] W.Q. Bai, H.Y. Huang, Y. Li, H.Y. Zhang, B. Liang, R. Guo, L.L. Du, Z.W. Zhang, Direct preparation of well-dispersed graphene/gold nanorod composites and their application in electrochemical sensors for determination of ractopamine, Electrochim. Acta 117 (2014) 322

[32] T.C. Tsai, F.H. Huang, J.J.J. Chen, Selective detection of dopamine in urine with electrodes modified by gold nanodendrite and anionic self-assembled monolayer, Sens. Actuators B 181 (2013) 179.

[33] M.Y. Lin, H.T. Han, D.W. Pan, H.Y.Zhang, Z.C. Su, Voltammetric determination of total dissolved iron in coastal waters using a glassy carbon electrode modified with reduced graphene oxide, Methylene blue and gold nanoparticles, Microchim. Acta 182 (2015) 805.

[34] G.H. Lu, X. Yao, X.G. Wu, T. Zhan, Determination of the total iron by chitosanmodified glassy carbon electrode, Microchem. J. 69 (2001) 81.

[35] M. Lu, R.G. Compton, Voltammetric determination of Iron(III) in water Electroanalysis 25 (2013) 1123. 